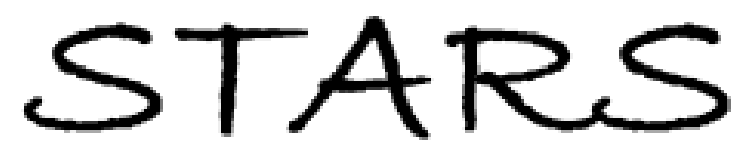

University of Central Florida

STARS

$1-1-2013$

\title{
Droplet deformation and manipulation in an electrified microfluidic channel
}

Jonathan D. Wehking

University of Central Florida

Larry Chew

University of Central Florida

Ranganathan Kumar

University of Central Florida

Find similar works at: https://stars.library.ucf.edu/facultybib2010 University of Central Florida Libraries http://library.ucf.edu

This Meeting Abstract is brought to you for free and open access by the Faculty Bibliography at STARS. It has been accepted for inclusion in Faculty Bibliography 2010 s by an authorized administrator of STARS. For more information, please contact STARS@ucf.edu.

\section{Recommended Citation}

Wehking, Jonathan D.; Chew, Larry; and Kumar, Ranganathan, "Droplet deformation and manipulation in an electrified microfluidic channel" (2013). Faculty Bibliography 2010s. 2576.

https://stars.library.ucf.edu/facultybib2010/2576

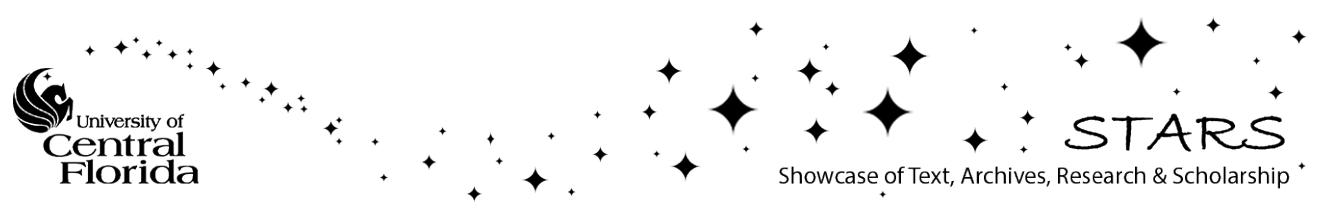




\section{Droplet deformation and manipulation in an electrified microfluidic channel}

Cite as: Appl. Phys. Lett. 103, 054101 (2013); https://doi.org/10.1063/1.4817008

Submitted: 09 May 2013 . Accepted: 12 July 2013 . Published Online: 30 July 2013

Jonathan D. Wehking, Larry Chew, and Ranganathan Kumar
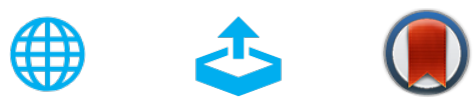

View Online

Export Citation

\section{ARTICLES YOU MAY BE INTERESTED IN}

Formation of dispersions using "flow focusing" in microchannels

Applied Physics Letters 82, 364 (2003); https://doi.org/10.1063/1.1537519

Effect of uniform electric field on the drop deformation in simple shear flow and emulsion shear rheology

Physics of Fluids 29, 072109 (2017); https://doi.org/10.1063/1.4995473

Effect of nonuniform electric field on the electrohydrodynamic motion of a drop in Poiseuille flow

Physics of Fluids 29, 052006 (2017); https://doi.org/10.1063/1.4983340

\section{Applied Physics Reviews} Now accepting original research 


\title{
Droplet deformation and manipulation in an electrified microfluidic channel
}

\author{
Jonathan D. Wehking, Larry Chew, and Ranganathan Kumar ${ }^{\mathrm{a})}$ \\ University of Central Florida, 4000 Central Florida Blvd, Orlando, Florida 32816, USA
}

(Received 9 May 2013; accepted 12 July 2013; published online 30 July 2013)

\begin{abstract}
This letter studies electrically induced droplet deformation to predictably pin and route droplets in a T-junction microchannel. Droplets of various sizes are shown to deform differently and respond to steep electric potential gradients. The relative magnitudes of inertial and electric forces are responsible for droplet deceleration and pinning, and may be manipulated to route a droplet in either direction of a T-junction. (C) 2013 AIP Publishing LLC. [http://dx.doi.org/10.1063/1.4817008]
\end{abstract}

Droplet formation between two immiscible fluids in a microfluidic T-junction has been studied extensively to characterize the size, frequency, and formation of liquid droplets. ${ }^{1-6}$ This letter introduces the idea that these liquid droplet emulsions can be manipulated in an electric field to obtain various deformations which can then be redirected in a microchannel. When an electric field is applied to a stationary suspended droplet, it will deform into either a prolate spheroid (elongate in the direction parallel to the electric field) or an oblate spheroid (elongate in the direction perpendicular to the electric field). ${ }^{7-18}$ Active and passive dielectrophoresis (DEP) have been demonstrated to manipulate droplets by leveraging the force imbalance induced by differing dipole moments, ${ }^{10,19}$ or by a surface charge using capillary electrophoresis. ${ }^{20}$ Electrowetting on dielectric (EWOD) has also been used to manipulate droplet behavior by adjusting the contact angle with an electric field. ${ }^{21,22}$ In this letter, we show how the droplets can be manipulated by treating all electrical effects generically. We will show that deformation of droplets can be exploited in the microchannels by judicious placement of steep electric potential gradients, producing nonuniform electric fields that perform passive droplet routing. Such manipulation of droplet deformation has applications in ink-jet printing, heat exchanger design, and optical emulsification technologies. Controlled droplet routing is also beneficial in biomedical fields.

Droplet formation dynamics in a microfluidic T-junction is influenced by several competing forces which include viscous shear, interfacial, and upstream pressure forces. ${ }^{2}$ As the dispersed fluid begins to penetrate the continuous channel, it encounters a viscous shear stress induced by the continuous fluid, and while it remains a "thread" still attached to the dispersed channel, local pressure increases immediately upstream of the T-junction due to the blockage of the continuous channel. Resisting these effects is the pressure jump across the fluid interface due to the interfacial tension of the fluid pair. The continuous and dispersed fluid flow rates, viscosities, and the interfacial surface tension all influence how far into the continuous channel the dispersed droplet will travel before it detaches. The capillary number $\left(C a=\mu_{c} v_{c} / \gamma\right)$ characterizes droplet size/volume and formation, where $\mu_{c}$ and $v_{c}$ are the continuous fluid dynamic viscosity and average

\footnotetext{
${ }^{\text {a) }}$ Author to whom correspondence should be addressed. Electronic mail: ranganathan.kumar@ucf.edu
}

velocity, respectively, and $\gamma$ is the interfacial tension of the continuous/dispersed fluid pair. By controlling the flow rate ratio $\left(Q=Q_{d} / Q_{c}\right)$ and viscosity ratio $\left(\lambda=\mu_{d} / \mu_{c}\right)$ in a T-junction, one can control the drop size. ${ }^{4,6}$ The subscripts $d$ and $c$ refer to dispersed and continuous fluids, respectively.

The immiscible two-fluid system in a T-junction with an electric field may be modeled as follows. The normalized incompressible continuity equation is $\nabla \cdot\left(\rho^{*} \mathbf{v}^{*}\right)=0$, and the normalized momentum equation is given by

$$
\begin{aligned}
\rho^{*} \frac{\partial \mathbf{v}^{*}}{\partial t^{*}}+\rho^{*}\left(\mathbf{v}^{*} \cdot \nabla\right) \mathbf{v}^{*}= & -\nabla p^{*}+R e^{-1} \nabla \cdot \mu^{*}\left(\nabla \mathbf{v}^{*}+\left(\nabla \mathbf{v}^{*}\right)^{T}\right) \\
& +E u_{e}^{-1} \nabla \cdot \boldsymbol{\sigma}^{M^{*}}+W e^{-1} \kappa^{*} \delta^{*} \hat{\mathbf{n}} \\
& +F r^{-2} \rho^{*} \hat{\mathbf{n}}_{g}
\end{aligned}
$$

Gravitational forces in microfluidics are small (large $\left.F r=v_{c} / \sqrt{g w_{c}}\right)$ compared to other forces, and are neglected. The Reynolds number $\left(R e=\rho_{c} v_{c} w_{c} / \mu_{c}\right)$, represents the ratio of inertial forces to viscous forces. Other dimensionless numbers include the electric Euler number $\left(E u_{e}=\rho_{c} v_{c}{ }^{2} / E_{0}{ }^{2} \epsilon_{0} \epsilon_{c}\right)$, representing the ratio of inertial forces to electric forces, and the Weber number $\left(W e=\rho_{c} v_{c}^{2} w_{c} / \gamma\right)$, representing the ratio of inertial forces to interfacial forces. The superscript * denotes dimensionless quantities. $v_{c}, \rho_{c}, \mu_{c}, \epsilon_{c}$ refer to the continuous fluid average velocity, density, dynamic viscosity, and relative permittivity, respectively, and $w_{c}, E_{0}, g, \gamma$, and $\epsilon_{0}$ are the continuous channel width, applied electric field, gravity, interfacial tension, and the vacuum permittivity, respectively. $\sigma^{M^{*}}$ is the Maxwell stress component of the stress tensor. According to the leaky dielectric model, ${ }^{23}$ ohmic conduction causes charge to accumulate at the fluid interface, and the mismatch in charge creates a tangential stress that must be balanced. Hydrodynamics provides the balance, resulting in fluid motion. Dielectric stresses acting normal to the fluid interface are also present due to the mismatch of dipole moments between the two fluids. The steady potential distribution in the system is defined by the conservation of charge, $\nabla \cdot\left(\sigma^{*} \mathbf{E}^{*}\right)=0$, where $\mathbf{E}^{*}$ is the electric field and $\sigma^{*}$ is the fluid conductivity.

The conservative two-phase level set method ${ }^{24}$ utilizes the "front capturing" approach to track the fluid-fluid interface, and is implemented numerically using COMSOL Multiphysics $4.3{ }^{18}$ To prevent numerical discontinuities at the interface shared by the two fluids, all fluid properties are represented as follows (using the dimensionless density as an 
example): $\rho^{*}=\rho_{c}^{*}+\left(\rho_{d}^{*}-\rho_{c}^{*}\right) \phi . \phi$ is the level set function defined as $\phi=0$ in the continuous fluid and $\phi=1$ in the dispersed fluid. Equation (1) is normalized, but still requires the presence of dimensionless fluid properties, such as $\rho^{*}$, $\mu^{*}, \epsilon^{*}$, and $\sigma^{*}$ in order to physically track the interface and resulting flow in each of the respective fluids. Constant volumetric laminar flow rates are set at the continuous and dispersed inlet boundaries, and a constant pressure/zero viscous stress condition is set at the outlet boundary. A constant hydrophobic contact angle is used at the channel walls to avoid electrowetting angle saturation effects at higher electrical potentials. ${ }^{21}$ The mesh density is determined using the results of an unstructured mesh sensitivity study on a stationary deforming droplet (deformation results discussed later). For meshes between $\approx 900-100000$ elements, the results begin to stabilize in the $3000-5000$ element range to within $10 \%$ of accepted theoretical values. Therefore, the mesh density for our numerical models is verified to fall within this range before simulation to avoid unnecessary computational effort. These mesh densities agree well with those reported by Lin (4489 elements). ${ }^{18}$ Phase initialization always converged within 30 iterations, and during the transient portion of the solution, the time steps were dynamically adjusted to ensure convergence while using a tolerance of 0.001 for all dependent variables.

Theoretical studies by Reddy and Esmaeeli ${ }^{17}$ were considered to validate numerical stationary droplet deformation in a suspending medium under an applied electric field. The droplets were represented two-dimensionally as infinite liquid jets under a transversely applied electric field. The deformed droplet boundaries result from the balancing of normal and tangential boundary forces (between hydrodynamic and electric effects), and cause the droplet to take either a prolate or oblate shape, as shown in Fig. 1(a). The theoretical deformation for a two-dimensional stationary droplet using the leaky dielectric model is ${ }^{17}$

$$
D=\frac{C a_{e}}{3}\left[\frac{R^{2}+R+1-3 q}{(1+R)^{2}}\right],
$$

where $R$ and $q$ are given in Table I. $C a_{e}=\epsilon_{c} \epsilon_{0} E_{0}{ }^{2} r / \gamma$ is the electric capillary number which quantifies the ratio of electric forces to interfacial forces, where $\epsilon_{c}, E_{0}, r$, and $\gamma$ are the permittivity of the continuous liquid, applied electric field, undeformed droplet radius, and interfacial surface tension, respectively. The vacuum permittivity is given by $\epsilon_{0}=8.854 \times 10^{-12} \mathrm{~F} / \mathrm{m}$. Droplet deformations observed numerically can be quantified as $D=(L-B) /(L+B)$, where $L$ and $B$ are the deformed droplet dimensions in Fig. 1(a). Other parameters used for these simulations were the Reynolds number $(R e=1)$, capillary number $(C a=1)$, density ratio $\left(\rho_{d} / \rho_{c}=1\right)$, and viscosity ratio $\left(\mu_{d} / \mu_{c}=1\right)$. When the permittivity ratio $q<10.33$, the simulated droplets deform into prolate spheroids $(D>0)$, and when $q>10.33$, they deform into prolate spheroids $(D<0)$. These results agree excellently with Eq. (2), as shown in Fig. 1(b). Deviations at larger magnitudes of $q$ and $D$ have been reported in other numerical studies ${ }^{13,14,18}$ and are due to Eq. (2) being formulated based on the assumption of small deformations.
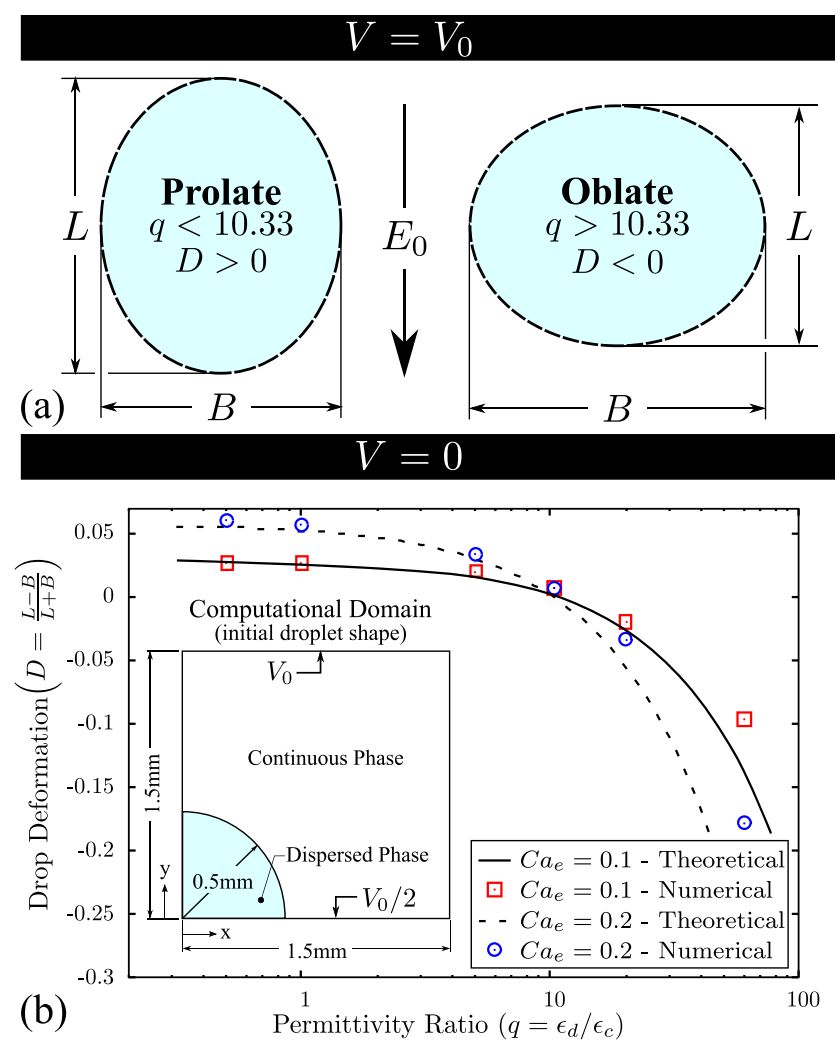

FIG. 1. (a) Schematic of two-dimensional stationary droplet deformed shapes. (b) Quantitative comparison of theoretical and numerical droplet deformations and computational domain (inset).

Flowing droplet simulations used a much more conductive dispersed liquid (deionized water) and a less conductive continuous liquid (silicone oil). The true conductivity ratio surpasses $R=10^{7}$, but is limited to $R=1000$ numerically to avoid divergence by attempting to resolve steep property gradients at the droplet boundary. This approximation is justified since droplet behavior is unaffected when one liquid's conductivity exceeds the other's by a factor of 100 or more. ${ }^{13}$ The density and viscosity used in simulating flowing droplets were $\rho_{c}=950 \mathrm{~kg} / \mathrm{m}^{3}$ and $\mu_{c}=0.019 \mathrm{~Pa} \cdot \mathrm{s}$ for silicone oil, and $\rho_{d}=1000 \mathrm{~kg} / \mathrm{m}^{3}$ and $\mu_{d}=0.001 \mathrm{~Pa} \cdot \mathrm{s}$ for deionized water. The inerfacial surface tension is $\gamma=0.045 \mathrm{~N} / \mathrm{m}$.

A smoothed step function of electrical potential was applied to the upper channel wall which resulted in droplet deceleration and pinning; this is explained in detail later. It is important to understand the relative magnitudes of the forces that would allow droplet deceleration and pinning within bulk continuous fluid flow. The inertial hydrodynamic force that allows the drop to flow down the channel is horizontal, but a dielectric force that contains non-uniform horizontal and vertical components is acting everywhere normal to the droplet surface, and always from the fluid with higher relative permittivity on the fluid with lower relative permittivity. The

TABLE I. Electrical properties used in simulations.

\begin{tabular}{lcc}
\hline \hline $\begin{array}{l}\text { Case } \\
(-)\end{array}$ & $\begin{array}{c}\text { Conductivity ratio } \\
\left(R=\sigma_{d} / \sigma_{c}\right)\end{array}$ & $\begin{array}{c}\text { Permittivity ratio } \\
\left(q=\epsilon_{d} / \epsilon_{c}\right)\end{array}$ \\
\hline Stationary droplets & 5 & See Fig. 1(b) \\
Flowing droplets & 1000 & $80 / 2.2$ \\
\hline \hline
\end{tabular}



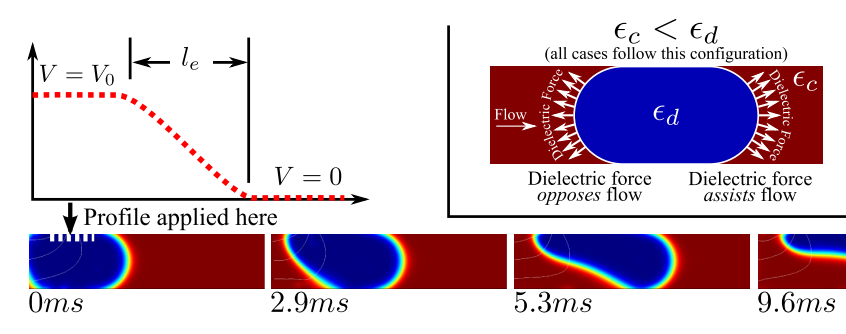

opposes flow

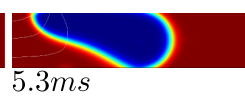

$9.6 m s$

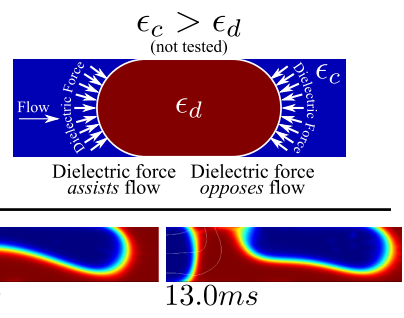

FIG. 2. Top right inset: Schematic of dielectric force on a flowing droplet. All flowing droplet simulations follow $\epsilon_{c}<\epsilon_{d}$ (left schematic of inset). Below and at left: Two-dimensional numerical results demonstrating droplet pinning, with a plot of the electrical potential boundary condition imposed at the top wall. top right inset of Fig. 2 illustrates how the dielectric force can both assist and oppose the inertial force, which leads to droplet deceleration and pinning. From Eq. (1), $E u_{e}$ emerges as a coefficient to the electric body force (term containing $\sigma^{M^{*}}$ ), and quantifies the ratio of inertial to electric forces. If the electric field, $E_{0}=V_{0} / l_{e}$, is defined as the gradient in the electrical potential step applied to the upper channel wall (Fig. 2, at left), simulations indicate that there exists a critical electric Euler number $\left(E u_{e}{ }^{*}\right)$ that characterizes droplet pinning. $l_{e}$ is the upper wall distance over which the electrical potential step occurs. Below $E u_{e}{ }^{*}$, the drop will be pinned to the wall; above $E u_{e}{ }^{*}$, the drop will flow along the channel. $E u_{e}{ }^{*}$ is also dependent on the undeformed drop diameter (d), as shown in Fig. 3. For $d^{*} \leq 1$, the droplet is pinned to the channel wall at $E u_{e}{ }^{*} \approx 0.82$. For $d^{*}>1$, the larger droplet volume (and subsequent mass) will increase the droplet's inertia, requiring the electric force necessary for pinning to increase (thus decreasing $E u_{e}{ }^{*}$ ). Other parameters causing the inertial force to increase (i.e., increase in flow rate or density) will require that the electric force (i.e., voltage) also increase in order to obtain the critical $E u_{e}{ }^{*}$ necessary for pinning. The curve fit $E u_{e}{ }^{*}=0.83-1.44\left(d^{*}-0.78\right)^{3}$ is consistent with a cubic dependency on the drop diameter, suggesting a linear dependency on droplet volume. Using an order of magnitude analysis, the ratio of inertial forces $\left(O \sim d^{3}\right)$ to electrical forces $(O \sim 1)$ provides physical consistency with the cubic dependency. When the droplet size increases, its larger surface area will experience an increase in viscous drag $\left(O \sim d^{2}\right)$, however, in the order of magnitude analysis, we have neglected this force.

To validate the two-dimensional numerical model without electrical effects, an experiment was devised in an unelectrified T-junction (Fig. 4, Configuration 1). A microfluidic T-junction test chip, $25 \mathrm{~mm} \times 50 \mathrm{~mm}$ in size, was

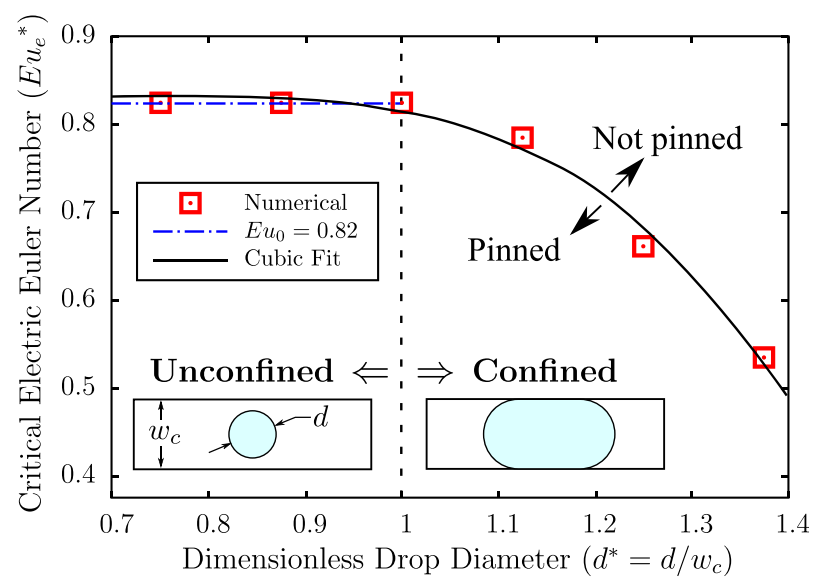

FIG. 3. Critical pinning electric Euler number dependency on drop diameter, highlighting confined and unconfined droplet regions. Cubic fit $R^{2}=0.989$. fabricated using poly(dimethylsiloxane) (PDMS) via soft lithography. The continuous channel width is $w_{c}=200 \mu \mathrm{m}$, $w_{d} / w_{c}=0.5$ and channel height $h=100 \mu \mathrm{m}$. Syringe pumps were used to drive the continuous (silicone oil) and dispersed (deionized water) fluids through their respective branches of the T-junction. The chip was placed under a standard laboratory microscope fitted with $4.0 \times$ magnification and Prosilica cameras sampling at 200 frames per second (fps) for visualization. For a continuous flow rate of $Q_{c}=200 \mu \mathrm{L} / \mathrm{h}$ and a dispersed flow rate of $Q_{d}=100 \mu \mathrm{L} / \mathrm{h}$, our numerical model predicted a drop length of $392 \mu \mathrm{m}\left(d^{*}=1.75\right)$ and a frequency of $4.2 \mathrm{drops} / \mathrm{s}$, for a deviation of $18 \%$ and $2.4 \%$, respectively, from our experiments.

A second experiment was devised to qualitatively validate the numerical model with electrification (Fig. 4, Configuration 2). The same microchannel is used as in Configuration 1, and a channel length of $10 \mathrm{~mm}$ after the $\mathrm{T}$-junction allowed for adequate imaging and electrode placements. Single strand 24 AWG copper wire $(\approx 0.5 \mathrm{~mm}$ diameter) acted as the electrodes, and were placed directly in the bulk PDMS material according to the schematic shown in Fig. 4. This placement of electrodes created steep potential gradients along the upper channel wall at locations in the vicinity of the electrodes. The resulting droplet behavior exhibits single-wall deceleration and pinning at approximately the same wall location for each passing droplet, similar to the droplet behavior demonstrated in our numerical models. The DC voltage applied to the electrodes was set to
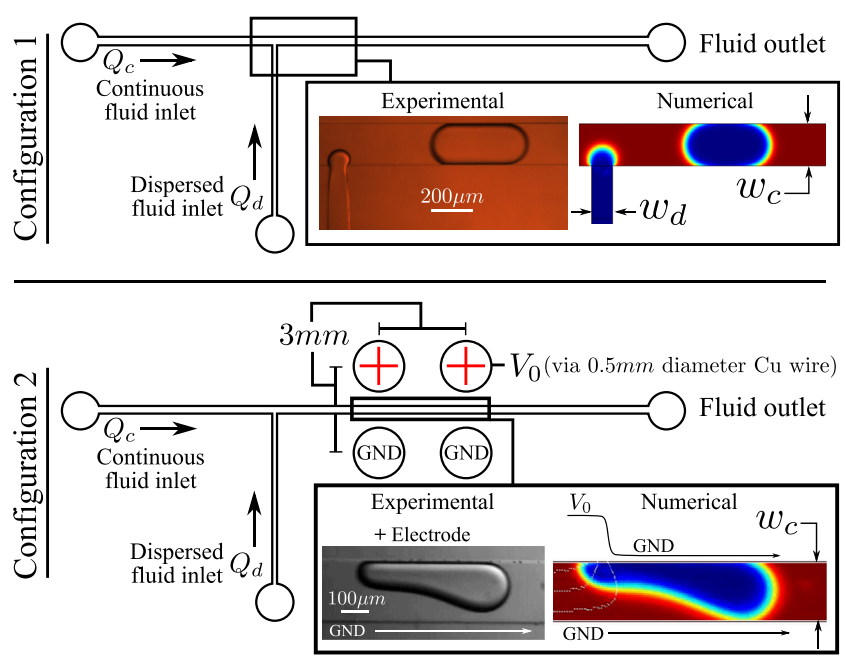

FIG. 4. Two configurations tested with their respective experimental and numerical droplet behavior. Configuration $1\left(d^{*}=1.75\right)$ was used to validate the two-phase numerical model by simulating droplet formation in an unelectrified T-junction. Configuration $2\left(d^{*}=1.6\right)$ was used to demonstrate electrically induced droplet pinning downstream of the T-junction. In configuration 2, the attenuated experimental potential is $V_{0} \approx 200 \mathrm{~V}$, and the applied numerical potential is $V_{0} \approx 390 \mathrm{~V}$. 


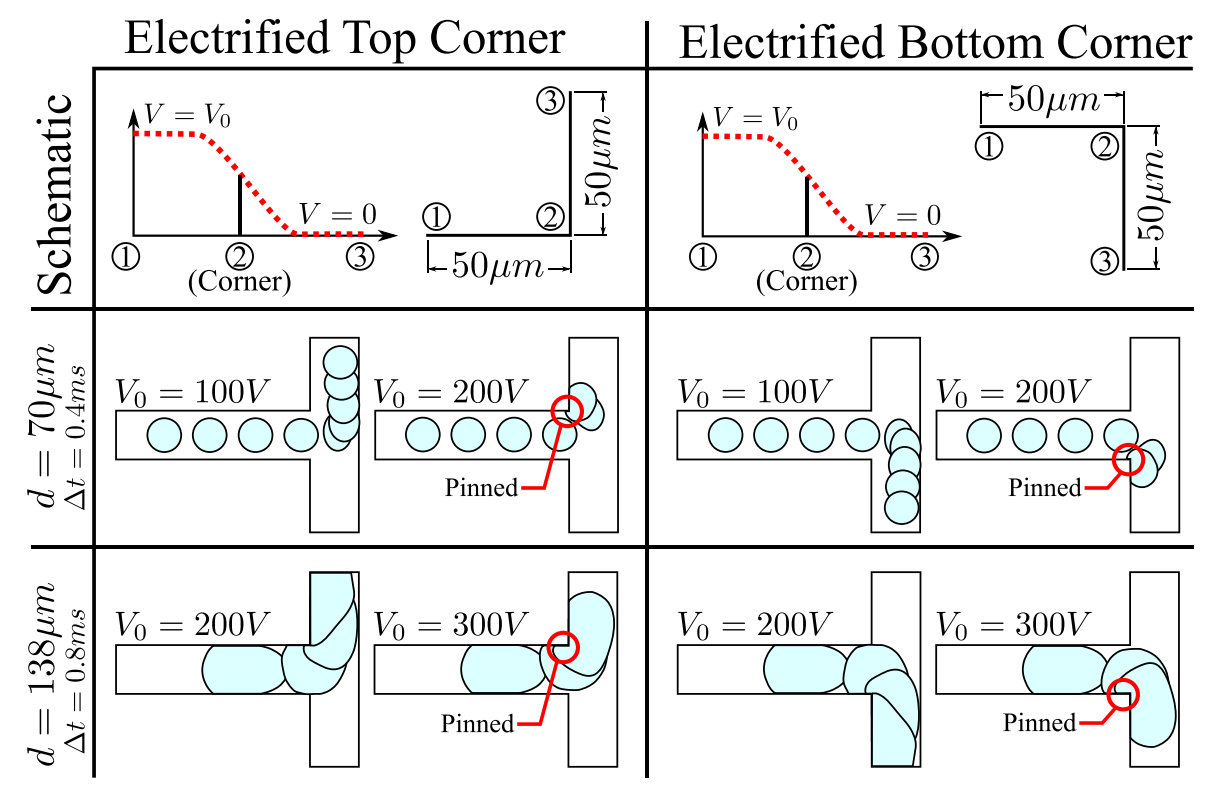

FIG. 5. Numerical simulation of droplet routing configurations. Schematic and sequence of images at different times for a T-junction electrified in the top corner (left column), and the bottom corner (right column) for two different drop diameters $(d)$. Once pinned, the droplet position at all future time steps overlap the final pinning location (droplet totally arrested with no additional deformation). The channel width is $100 \mu \mathrm{m}$, and fluid properties are identical to those listed previously.
$V_{0}=2000 \mathrm{~V}$, and the pinning locations were located within $1.0 \mathrm{~mm}$ upstream and/or downstream of each electrode pair for a droplet of diameter $d^{*}=1.6$. The copper wire electrodes were spaced approximately $1.5 \mathrm{~mm}$ from the microchannel, causing the applied voltage to suffer significant attenuation (due to the relatively inert electrical properties of the bulk PDMS material) before reaching the flowing droplets inside the microchannel. Three-dimensional electrostatic models performed in COMSOL Multiphysics indicate $\mathrm{a} \approx 90 \%$ reduction in applied voltage under these circumstances, bringing the experimental voltage drop along the microchannel boundaries to $V_{0} \approx 200 \mathrm{~V}$. The numerically applied voltage required for similar pinning behavior (under nearly identical conditions) was $V_{0} \approx 390 \mathrm{~V}$. The experimental voltage required for droplet pinning is smaller in magnitude than the voltage required numerically, but the agreement is still good considering the two-dimensional numerical approximation and the crude positioning of the copper wire electrodes.

We can exploit these pinning phenomena by applying steep gradients in electrical potential numerically at specific locations within a microchannel, thus allowing for increased control over flowing liquid droplet emulsions. A simplified Tjunction configuration is numerically simulated for two different diameter droplets: $d=70 \mu \mathrm{m}$, and $d=138 \mu \mathrm{m}$ (refer to Fig. 5). A steep electrical potential gradient is applied at either the upper or lower corner in order to route a droplet into either the upper or lower branch of the T-junction microchannel. Passive droplet routing is possible in this configuration and is attributed to identical phenomena as seen with pinning. As a flowing droplet approaches the T-junction, the dielectric force caused by the steep electrical potential gradient in the corner begins to decelerate the droplet on one side. As the droplet decelerates, the viscous shear induced by the continuous flow causes the droplet to turn into the upper part of the T-junction, effectively pivoting around the electrified corner. The critical electrical potential for pinning the smaller drop at the corner is between $100 \mathrm{~V}$ and $200 \mathrm{~V}$, while the critical electrical potential for pinning the larger drop is between $200 \mathrm{~V}$ and $300 \mathrm{~V}$, suggesting the effects are also dependent on drop size under these flow conditions (same trend as Figure 3). This behavior is consistent for flow situations according to the following set of parameter values: Reynolds number $\left(\operatorname{Re}=\rho_{c} v_{c} w_{c} / \mu_{c} \approx 1\right)$, Froude number $\left(F r=v_{c} / \sqrt{g w_{c}} \approx 6\right.$. $)$, Weber number $\left(W e=\rho_{c} v_{c}{ }^{2} w_{c} / \gamma \approx 0.1\right)$, capillary number $\left(C a=\mu_{c} u_{c} / \gamma \approx 0.1\right)$, and electric capillary number $\left(C a_{e}=\epsilon_{0} \epsilon_{c} E_{0}^{2} r / \gamma \approx 0.2\right)$.

In conclusion, droplet deformations were exploited to perform actuation in a T-junction microchannel. Such routing was accomplished by utilizing the inherent electrical properties of the fluid pair without the need for any additives to enhance deformations caused by an electric field. The smoothed step function along a channel boundary was required to manipulate the droplets, but needed to be adjusted such that the electrical forces were large enough to pull the droplets in a preferential direction of the T-junction without permanently halting their continued progress. The behavior also demonstrates consistency with a cubic dependency on the droplet diameter. In addition to traditional mechanical applications such as enhanced spatial control (i.e., ink-jet printing), micropump fabrication, or electrohydrodynamic (EHD) enhanced heat transfer, this physical phenomenon has tremendous potential in biochemical analyses, such as electrokinetic assays and miniturized total analysis systems ( $\mu$ TAS). Precision control of high throughput droplet microreactors is also very attractive in colloidal crystallization and biomedical applications. This study paves the way for microchannel designers in any field to generate droplets of desired size and passively route them in sub-microchannels.

${ }^{1}$ T. Thorsen, R. W. Roberts, F. H. Arnold, and S. R. Quake, Phys. Rev. Lett. 86, 4163 (2001).

${ }^{2}$ P. Garstecki, M. J. Fuerstman, H. A. Stone, and G. M. Whitesides, Lab Chip 6, 437 (2006).

${ }^{3}$ M. De Menech, P. Garstecki, F. Jousse, and H. A. Stone, J. Fluid Mech. 595, 141 (2008).

${ }^{4}$ A. Gupta, S. M. S. Murshed, and R. Kumar, Appl. Phys. Lett. 94, 164107 (2009).

${ }^{5}$ A. Gupta and R. Kumar, Microfluid. Nanofluid. 8, 799 (2010).

${ }^{6}$ A. Gupta and R. Kumar, Phys. Fluids 22, 122001 (2010).

${ }^{7}$ S. Torza, R. G. Cox, and S. G. Mason, Philos. Trans. R. Soc. London, Ser. A 269, 295 (1971).

${ }^{8}$ O. Vizika and D. A. Saville, J. Fluid Mech. 239, 1 (1992). 
${ }^{9}$ M. Sato, S. Kato, and M. Saito, IEEE Trans. Ind. Appl. 32, 138 (1996).

${ }^{10} \mathrm{P}$. Singh and N. Aubry, Electrophoresis 28, 644 (2007).

${ }^{11}$ J. D. Sherwood, J. Fluid Mech. 188, 133 (1988).

${ }^{12}$ J. Zhang and D. Y. Kwok, J. Comput. Phys. 206, 150 (2005).

${ }^{13}$ J. Q. Feng and T. C. Scott, J. Fluid Mech. 311, 289 (1996).

${ }^{14}$ J. Hua, L. K. Lim, and C.-H. Wang, Phys. Fluids 20, 113302 (2008).

${ }^{15}$ J. M. Lopez-Herrera, S. Popinet, and M. A. Herrada, J. Comput. Phys. 230, 1939 (2011)

${ }^{16}$ A. Fernandez and G. Tryggvason, Phys. Fluids 17, 093302 (2005).
${ }^{17}$ M. N. Reddy and A. Esmaeeli, Int. J. Multiphase Flow 35, 1051 (2009).

${ }^{18}$ Y. Lin, Electrophoresis 34, 1 (2013).

${ }^{19}$ K. Ahn, C. Kerbage, T. P. Hunt, R. M. Westervelt, D. R. Link, and D. A. Weitz, Appl. Phys. Lett. 88, 024104 (2006).

${ }^{20}$ V. Dolnik, S. Liu, and S. Jovanovich, Electrophoresis 21, 41 (2000).

${ }^{21}$ F. Mugele and J.-C. Baret, J. Phys.: Condens. Matter 17, R705 (2005).

${ }^{22}$ K. Mohseni and A. Dolatabadi, Ann. N.Y. Acad. Sci. 1077, 415 (2006).

${ }^{23}$ J. R. Melcher and G. I. Taylor, Annu. Rev. Fluid Mech. 1, 111 (1969).

${ }^{24}$ E. Olsson, G. Kreiss, and S. Zahedi, J. Comput. Phys. 225, 785 (2007). 